\title{
BMJ Open Effect of Community ART Groups on retention-in-care among patients on ART in Tete Province, Mozambique: a cohort study
}

\author{
Tom Decroo, ${ }^{1}$ Barbara Telfer, ${ }^{1}$ Carla Das Dores, ${ }^{2}$ Richard A White, ${ }^{3}$ \\ Natacha Dos Santos, ${ }^{1}$ Alec Mkwamba, ${ }^{1}$ Sergio Dezembro, ${ }^{1}$ Mariano Joffrisse, ${ }^{1}$ \\ Tom Ellman, ${ }^{4}$ Carol Metcalf ${ }^{4}$
}

To cite: Decroo T, Telfer B, Dores CD, et al. Effect of Community ART Groups on retention-in-care among patients on ART in Tete Province, Mozambique: a cohort study. BMJ Open 2017;7:e016800. doi:10.1136/ bmjopen-2017-016800

- Prepublication history for this paper is available online. To view these files, please visit the journal online (http://dx.doi org/10.1136/bmjopen-2017016800).

Received 13 March 2017 Revised 8 June 2017 Accepted 30 June 2017

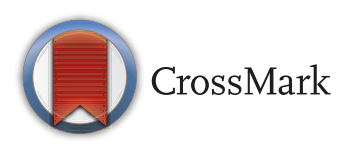

${ }^{1}$ Mission Mozambique Médecins Sans Frontières, Operational Center Brussels, Tete, Mozambique

${ }^{2}$ Direcção Provincial de Saúde, Ministério da Saude de Moçambique, Tete, Moçambique ${ }^{3}$ Department of Infectious Disease Epidemiology and Modelling, Norwegian Institute of Public Health, Oslo, Norway ${ }^{4}$ Southern Africa Medical Unit, South Africa, Médecins Sans Frontières, Operational Center Brussels, Cape Town, South Africa

Correspondence to Dr Tom Decroo; tomdecro02@gmail.com

\section{ABSTRACT}

Objectives Estimate the effect of participation in Community ART Groups (CAG) versus individual care on retention-in-care (RIC) on antiretroviral therapy (ART). Design Retrospective cohort study.

Setting High levels of attrition (death or loss-to-follow-up (LTFU) combined) on ART indicate that delivery models need to adapt in sub-Saharan Africa. In 2008, patients more than 6 months on ART began forming CAG, and took turns to collect ART refills at the health facility, in Tete Province, Mozambique,

Participants 2406 adult patients, retained in care for at least 6 months after starting ART, during the study period (date of CAG introduction at the health facility-30 April 2012).

Methods Data up to 30 April 2012 were collected from patient records at eight health facilities. Survival analysis was used to compare RIC among patients in CAG and patients in individual care, with joining a CAG treated as an irreversible time-dependent variable. Multivariable Cox regression was used to estimate the effect of CAG on RIC, adjusted for age, sex and health facility type and stratified by calendar cohort.

Results 12-month and 24-monthRIC from the time of eligibility were, respectively, $89.5 \%$ and $82.3 \%$ among patients in individual care and $99.1 \%$ and $97.5 \%$ among those in CAGs $(p<0.0001)$. CAG members had a greater than fivefold reduction in risk of dying or being LTFU (adjusted HR: $0.18,95 \% \mathrm{Cl} 0.11$ to 0.29 ).

Conclusions Among patients on ART, RIC was substantially better among those in CAGs than those in individual care. This study confirms that patient-driven ART distribution through CAGs results in higher RIC among patients who are stable on ART.

\section{INTRODUCTION}

Currently, an estimated 36.7 million people are living with HIV (PLHIV), of whom 17 million were on antiretroviral therapy (ART) at the end of 2015. ${ }^{1}$ The WHO endorses the 90-90-90 Joint United Nations Programme on HIV/AIDS targets: by 2020 , $90 \%$ of people living with HIV should know
Strengths and limitations of this study

- Community ART Groups (CAG) were piloted first in Tete Province, Mozambique. The effect of participation in CAG versus individual care on retention-in-care (RIC) on ART was not yet assessed in this pilot project.

- A large number of patients, with diverse characteristics, were included in the analysis. The findings are representative of 'real-life' programmatic conditions.

- Another strength is that through our methodological approach, we minimised the potential for survival bias by (1) starting follow-up 6 months after ART initiation to exclude patients who had not yet stabilised on ART and (2) treating CAG status as a time-dependent variable to ensure that RIC prior to joining a CAG was taken into account.

- However, the applied exclusion criteria may have resulted in some selection bias, making the findings less generalisable. Moreover, patients who opted to join a CAG and those who remained in individual care may have differed with respect to factors which we did not take into consideration in the analysis.

their HIV status. Of those, $90 \%$ should be on ART, and $90 \%$ of people on ART should be virologically suppressed. Or, when combined as a single indicator, $73 \%$ of all PLHIV should be virologically suppressed. ${ }^{2}$

Will it be feasible to achieve $73 \%$ of all PLHIV on ART and virologically suppressed by 2020 ? Such an unprecedented undertaking will require innovative approaches, especially in sub-Saharan Africa, where the HIV burden is the highest and health workforce gaps and other challenges hamper response. ${ }^{3}$ In addition, high levels of attrition (death or loss-to-follow-up (LTFU) combined) undermine the proven benefits of early treatment for individuals and the prevention of onward transmission of $\mathrm{HIV}^{4}$ A recent systematic 
review reported attrition rates in ART programme in African countries of $18 \%, 24 \%$ and $31 \%$ after 6 months, 1 year and 2 years of ART, respectively. ${ }^{4}$ Distance to health facilities, transport costs, long waiting times at the health facilities, work responsibilities and family commitments have been reported as reasons for defaulting treatment. ${ }^{5}$ ART delivery closer to patients' homes is effective at improving retention-in-care (RIC).$^{5}$

To enrol and retain millions of PLHIV on ART, health systems have had to adapt during the past decade. Several policies have been implemented to increase the capacity of understaffed health systems. Treatment has been decentralised from specialised HIV clinics to peripheral primary healthcare facilities. ${ }^{6}$ Tasks have been shifted from doctors to nurses, from nurses to lay health workers, and from lay health workers to patients. ${ }^{7}$ Additionally, in some countries delivery models have become increasingly patient-centred, allowing patients to combine lifelong ART refills with a normal social and economic life. ${ }^{58}$

Mozambique is one of the countries that have adopted a patient-centred ART delivery model. However, despite decentralisation of ART provision, starting in 2006, LTFU rates remained unacceptably high. One study showed an overall attrition rate of 37 per 100 person-years. ${ }^{9}$ Another Mozambican study showed that half of those who started ART were either dead or LTFU at 3 years follow-up. ${ }^{10}$ Strategies, such as home visits to patients LTFU, had been unsuccessful in bringing patients back to care. ${ }^{11}$ Patients reported long distances, lack of information, queuing at health facilities and stigma associated with regular clinic attendance, as barriers to RIC. ${ }^{12}$

To overcome these barriers associated with the standard, clinic-based, individual-care approach to ART delivery and drawing on published accounts of patient involvement in chronic disease care, ${ }^{13}$ the Health Directorate of Tete Province and Médecins Sans Frontières proposed that clinically stable patients on ART be given the option of forming peer groups and becoming involved in ART delivery and monitoring.

Patients on ART are given the option of joining a peer group, or remaining in clinic-based individual care, and can move between the two models of care, according to their preference. These peer groups are named Community ART Groups (CAGs). Giving patients a high level of autonomy, the CAG model is the most patient-driven, community-based ART delivery model described to date. ${ }^{14}$ Lay counsellors played an important role in forming and monitoring CAGs. ${ }^{15}$ The CAG model has previously been described in more detail. ${ }^{16}$

Four-year RIC was $92 \% .{ }^{17}$ Despite this high RIC on ART among patients in CAGs, these previous studies did not assess the relative effectiveness of the CAG model and the standard, clinic-based, individual care approach in retaining patients on ART, in Tete province, where CAG were piloted. We conducted a study to estimate the effect of the CAG model relative to standard individual care on RIC among patients on ART.

\section{METHODS}

\section{Study design}

We conducted a retrospective cohort study using programme data.

\section{Study setting}

Mozambique has a population of 23.9 million inhabitants, of whom more than $70 \%$ live in rural areas. ${ }^{18} \mathrm{HIV}$ prevalence among sexually active people is estimated to be $10.5 \%$. Over 1.5 million people in Mozambique are living with HIV. ${ }^{19}$ The government began providing ART in 2003. ${ }^{20}$ By the end of 2015, ART coverage was about $53 \% .^{19}$

The rural Province of Tete, in Mozambique, has 105 health facilities, spread across 15 districts. By mid-2012 only 32 of the $105(30.5 \%)$ facilities in Tete Province offered ART. ${ }^{20}$ Decentralisation of ART provision towards peripheral clinics to increase accessibility of ART has been hampered by infrastructural constraints, a shortage of medically qualified staff, organisational challenges and a lack of regulation to push for task shifting from nurses to lay health-workers. ${ }^{21}$

\section{CAGs}

CAGs are peer groups in which members take turns to travel to the clinic to collect monthly ART refills for all group members. To join a CAG, patients are required to be at least 15 years old, and to have been on ART for at least 6 months and to be stable on treatment. Each CAG has a maximum of six members. Members take turns to travel to the clinic to collect monthly ART refills for all group members. Every month, before the CAG representative attends the health facility to collect the ART refills, the group meets in their community to discuss each member's current health and treatment status and any travel plans. The CAG representative whose turn is to collect the monthly ART refills has a clinical consultation and reports on the status of the other group members (retained on ART in the group, died, travelled, etc). This information is recorded on a group monitoring card, which is kept in the clinic and updated each month. The group monitoring card includes the name of the CAG, the names of the CAG members, their ART regimen and the monthly pill count. ${ }^{16}$ CAG members are advised to make unscheduled visits to the health facility between ART refill appointments if they develop health problems, as do other patients who develop health problems during the intervals between scheduled appointments.

Of the 32 facilities in Tete Province that were providing ART in 2012, $12(37.5 \%)$ implemented the CAG model in 2008 or 2009. Differences in the management of patients in standard individual care and those in CAGs are summarised in table 1 .

\section{Study sites and population}

Of 12 health facilities that had implemented the CAG model by the end of 2009, eight (Manje, Changara, Songo, Chitima, Mutarara, Moatize, Zobue and Boroma) 


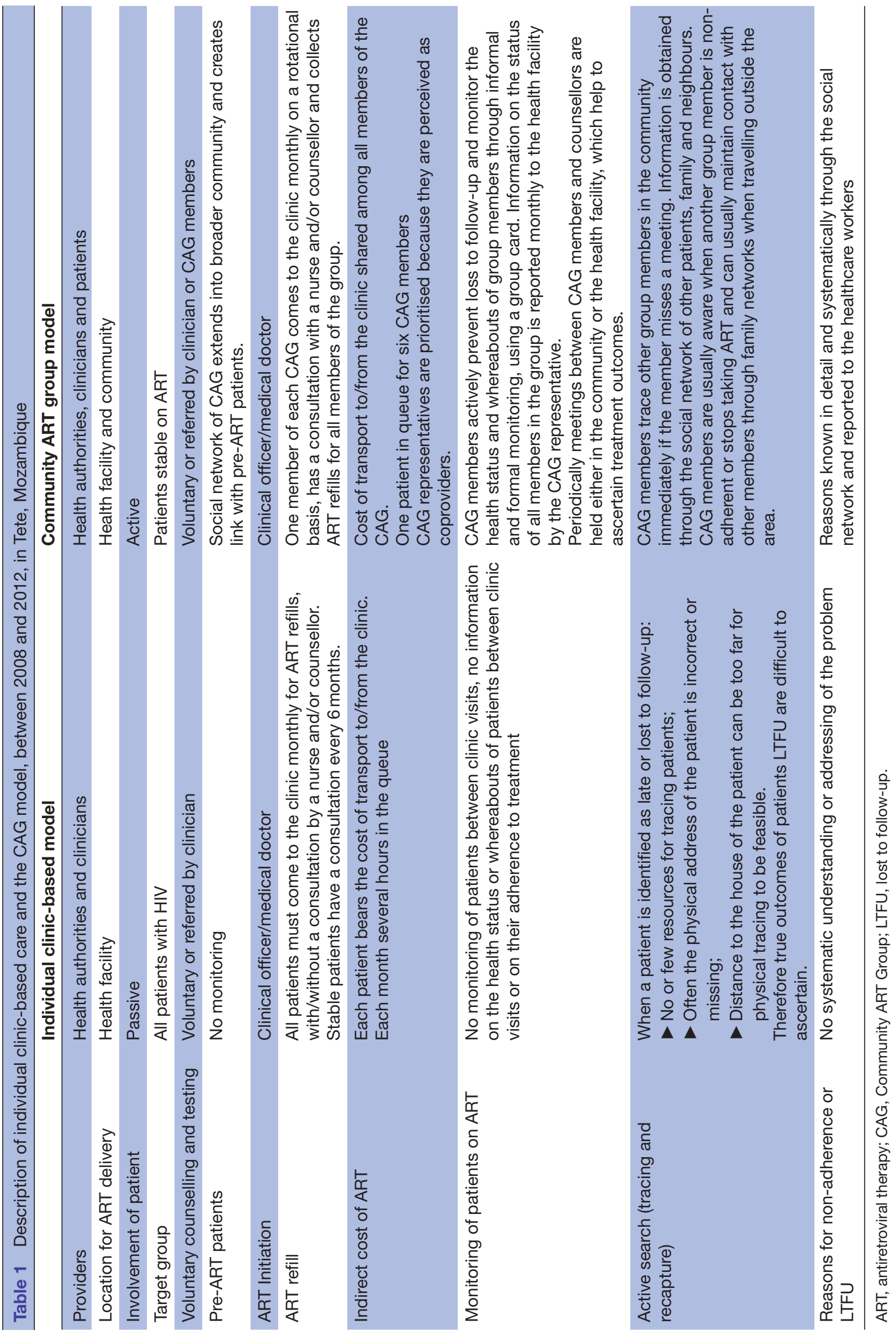


were included in this study. The other four facilities were excluded because the majority of patients on ART $(>80 \%)$ were enrolled in CAGs, leaving few patients in standard individual care to serve as a comparison group.

Patients included in the study were known to be 15 to 59 years of age at ART commencement and had started ART six or less months prior to or after the CAG model was introduced at the health facility. To minimise survival bias, patients who started ART more than 6 months before the CAG model was introduced at the health facility that they were attending and patients who transferred to the health facility more than 6 months after starting ART were excluded from the analysis. Patients younger than 15 years, 60 years and older, with an unknown age at ART initiation, were also excluded from the analysis. Patients who remained in care for less than 6 months after starting ART were excluded because patients are required to be stable on ART to be eligible to join a CAG, and mortality is highest in the first 6 months after starting ART. ${ }^{22} 23$

\section{Study period}

The start of the study period varied by health facility, starting on the date that the first CAGs were formed at the facility. The CAG starting dates were respectively 23 August 2008, 24 September 2008, 10 October 2008, 8 January 2009, 13 May 2009, 15 September 2009, 16 September 2009 and 14 July 2009 for health facility Zobue, Manje, Changara, Boroma, Moatize, Songo, Mutarara and Chitima. Patients at all eight study facilities were followed-up until the end of April 2012. For the purpose of this analysis, patients entered the cohort on the date on which they became eligible to join a CAG, defined as 6 months after starting ART.

\section{Data collection and definition of variables}

Patient-files and clinic-held copies of CAG cards were used as data sources. Data were abstracted during the second half of 2012 and 2013 and entered into a Microsoft Access database. CAG monitoring tools and processes have been described elsewhere. ${ }^{16}$

The information collected included patient sociodemographic characteristics (sex, age at ART initiation, date of ART initiation, CD4 results, date of joining a CAG and date of returning to individual care, if applicable), treatment outcomes and dates. For patients in CAG, the source for the treatment outcome and date was the CAG card and the patient-file. All other variables were solely retrieved from the patient-files. The following treatment outcomes were recorded: retained in care at the end of the study period (30 April 2012), dead, lost to follow-up (LTFU) and transferred out. LTFU was defined as being more than 2 months overdue for the most recent appointment or scheduled ART refill. Similarly, CAG members who did not collect the scheduled ART refill within their CAG were defined as LTFU. Health facilities were categorised as periurban or rural based on the geographical setting in which they are located. The two periurban facilities (Moatize and Songo) have medical specialists, a referral laboratory and radiology facilities available, and the rural facilities (Manje, Changara, Chitima, Mutarara, Zobue and Boroma) are primary healthcare facilities run by nurses.

\section{Data analysis}

The analysis was performed using Stata V.14 (StataCorp).

Some numeric variables were categorised to facilitate the analysis. Median and IQRs were calculated for numeric variables and proportions for categorical variables.

Survival analysis was used to compare RIC among patients in CAG and patients in individual care. Joining a CAG was treated as an irreversible time-dependent variable. Patients were 'not in a CAG', until they joined a CAG and 'in a CAG' from the date that they joined a CAG. CAG members who returned to individual care $(n=11)$ were retained in the CAG group in the survival analyses. Univariable and multivariable Cox regression were used to estimate crude HRs and adjusted HRs (aHRs) for attrition. The aHRs were adjusted for age, sex and health facility type and stratified by cohort (calendar period of ART initiation by 6-month intervals). Cohorts were restricted to 2010 to allow for at least 12 months follow-up.

Patients who remained in care at the end of the study period had their follow-up censored on 30 April 2012. Patients who were LTFU, or who died during the study period, were considered as having experienced the outcome event (attrition), with the outcome date defined as the most recent date of contact with the health facility, either in the form of an individual clinic visit or an ART refill collected by another CAG member on the patient's behalf. Patients who were transferred to another facility were censored on the date of transfer.

\section{Ethics}

This study was approved by the Ethics Review Board of Médecins Sans Frontières (Geneva, Switzerland) and the Mozambican National Bioethics Committee (Comite Nacional De Bioetica para a Saúde).

\section{RESULTS}

During the study period, between 1 February 2008 and 30 April 2012, 9266 patients were provided with ART in the eight health facilities. Of these patients, 2406 were included in the analysis and 6860 were excluded for reasons shown in figure 1.

Of the 2406 patients who satisfied the inclusion criteria, $901(37.5 \%)$ joined a CAG during the study period (table 2). Patients who joined a CAG were also more likely to be female (CAG: 70.3\%; 631/901; non-CAG: 59.9\%; 883/1505) and attending a rural clinic (CAG: 64.8\%; 584/901; non-CAG: 57.3\%; 862/1505). Patients who joined a CAG had a longer follow-up time (median: 26 months; IQR: 18 to 33 months) from the date that they entered the cohort and the end of the study than those 


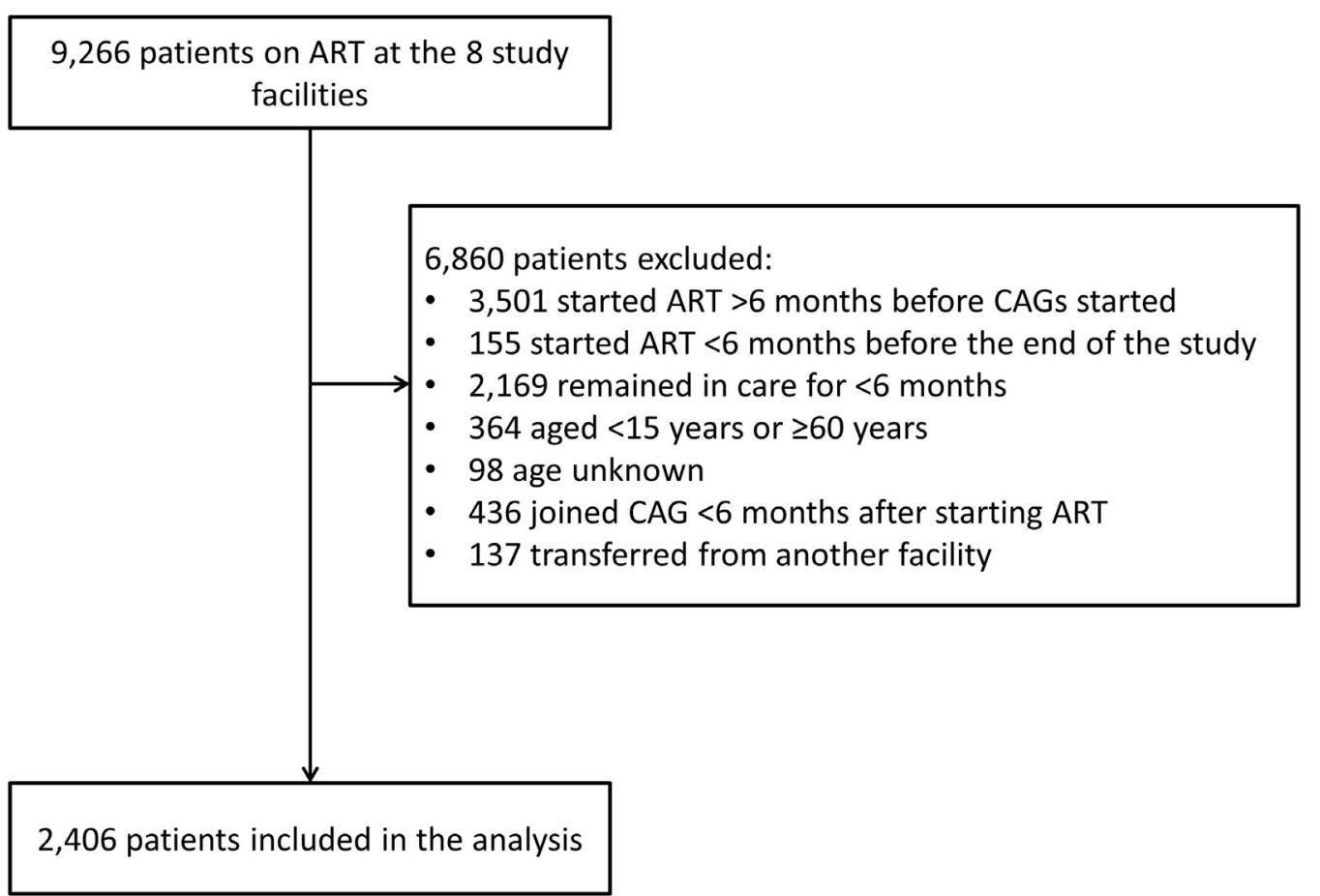

Figure 1 Study flow diagram: inclusion of patients on ART in the study.(Uploaded separately). ART, antiretroviral therapy; CAG, Community ART groups.

who did not join a CAG (median: 16 months; IQR: 7 to 27 months).

CAG patients joined a CAG after a median of 8.3 (IQR 3.6 to 16.7 ) months from the time of eligibility ( 6 months after starting ART). Overall, 279 out of 2406 (12\%) patients died or were LTFU by the end of the study period.

Overall, 12 month RIC from the date of eligibility was $90.8 \%$ (95\% CI89.5\% to 92.0\%) and 24-month RIC was $86.0 \%$ (95\% CI $84.2 \%$ to $87.6 \%$ ). RIC was significantly greater among patients in CAGs than those not in CAGs (stratified log-rank test: $\mathrm{p}<0.0001$ ) (figure 2). Twelvemonth RIC was $99.1 \%$ (95\% CI $97.3 \%$ to $99.7 \%$ ) among those in CAGs and $89.5 \%$ (95\% CI : $87.9 \%$ to $90.8 \%$ ) among those not in CAGs (table 3).

Adjusted for age, gender, health facility type and stratified by calendar period of ART initiation, patients in CAG had a more than fivefold lower rate of attrition (aHR: 0.18,
95\% CI 0.11 to 0.29 ) (table 3 ). The risk of attrition was higher among patients younger than 25 years compared with those aged 30-39 years (aHR: 1.65 , 95\% CI 1.17 to 2.32) and among males compared with females (aHR: $1.80,95 \%$ CI 1.41 to 2.30 ) (table 3 ).

\section{DISCUSSION}

We found that RIC among patients in CAGs was substantially higher than among patients in individual care. After adjustment for age, gender, health facility type and after stratification by calendar period of ART initiation, patients in CAG were more than five times less at risk to die or to be LTFU. Other studies on RIC in CAG and individual care showed similar findings. Reports of high RIC in CAG in Tete Province informed CAG pilots, in Mozambique and in Lesotho. The Mozambique national pilot showed

Table 2 Characteristics of patients included in the analysis, by CAG status

\begin{tabular}{llll}
\hline & Did not join a CAG & Joined a CAG & Total cohort \\
\hline $\begin{array}{l}\text { Total (n, column \%) } \\
\text { Sex * }(\mathrm{n}, \text { column \%) }\end{array}$ & $1505(100)$ & $901(100)$ & $2406(100)$ \\
$\quad$ Female & $883(59.9)$ & $631(70.3)$ & $1514(63.1)$ \\
$\quad$ Male & $617(41.1)$ & $267(29.7)$ & $884(36.9)$ \\
Age at ART initiation (years) (median, IQR) & $32(26-39)$ & $33(27-40)$ & $32(27-39)$ \\
Health facility type (n, column \%) & & $317(35.2)$ & $960(39.9)$ \\
$\quad$ Periurban & $643(42.7)$ & $584(64.8)$ & $1446(60.1)$ \\
$\quad$ Rural & $862(57.3)$ & & \\
\hline
\end{tabular}

*8 $(0.3 \%)$ patients did not have their sex recorded.

ART, antiretroviral therapy; CAG, Community ART Group. 


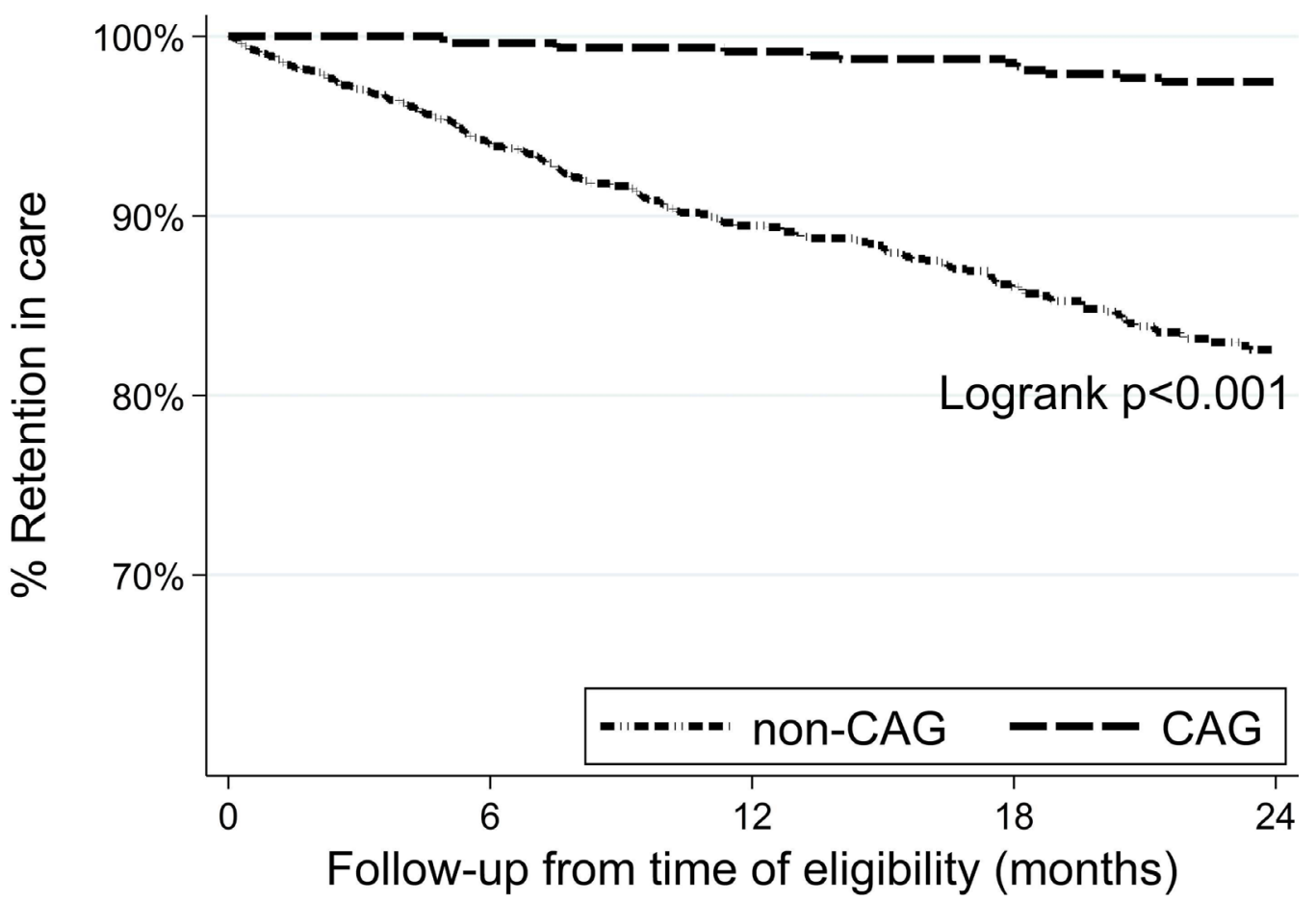

Figure 2 Retention-in-care by CAG status among 2406 patients on ART, between 2008 and 2012, in Tete, Mozambique. (Uploaded separately). ART, antiretroviral therapy; CAG, Community ART Group.

91.4\% and $82.9 \%$ RIC in CAG and individual care, respectively. This study included patients from 68 health facilities in seven different provinces (not Tete Province), a mix of urban and rural and high and low volume ART clinics. ${ }^{24}$ The Médecins Sans Frontières (MSF) supported pilot in Lesotho showed $98.7 \%$ and $90.2 \%$ RIC in CAG and individual care, respectively. ${ }^{25} \mathrm{CAG}$ members reported several benefits including time and cost savings. They reported that less frequent clinic visits was associated with reduced experiences of stigma in the community and viewed the $\mathrm{CAG}$ as a protective environment where they could share treatment experiences confidentially. ${ }^{1525}$

Overall, 12-month RIC from the date of eligibility (6 months after starting ART) was $90.8 \%$ and 24 month RIC was $86.0 \%$. These findings are similar to what is reported by other studies conducted in Mozambique. In a study conducted in rural Mozambique, 2-year attrition among patients more than 12 months on ART was $16.2 \% .{ }^{26}$ Another Mozambican study showed late attrition rates (after 6 months on ART) of 15 patients per 100 personyears in urban clinics and 23 patients per 100 person-years in rural clinics. ${ }^{9}$ A systematic review analysed data from eight Mozambican studies and found attrition of $17 \%$ at 6 months, $28 \%$ at 12 months and $44 \%$ at 24 months. ${ }^{4}$

A strength of this study is the large number of patients, with diverse characteristics included in the analysis. Another strength is that all the study facilities gave patients the option between individual, clinic-based care and CAG, thus enabling the models of care to be compared under 'real-life' programmatic conditions. Therefore, our findings are representative of the reality of the programme in Tete. Another strength is our methodological approach.
Patients entered the cohort after being on ART for 6 months, thus excluding patients who had not yet stabilised on ART. Among patients on ART, attrition has been found to be highest immediately after ART initiation, gradually declining over the following year. ${ }^{4}$ We minimised the potential for survival bias by excluding patients who had started ART more than 6 months before CAGs were introduced at the facility; starting follow-up 6 months after ART initiation to exclude patients who had not yet stabilised on ART; treating CAG status as a time-dependent variable to ensure that RIC prior to joining a CAG was taken into account and stratifying the Cox regression analyses by calendar period of entry into the cohort to take into account potential interaction between CAG status and calendar period with respect to attrition. Finally, we adhered to the Strengthening the Reporting of Observational Studies in Epidemiology guidelines for cohort studies.

However, there are also limitations to this study. The exclusion criteria that we chose may have resulted in some selection bias, making the findings less generalisable. Moreover, patients who opted to join a CAG and those who remained in individual care may have differed with respect to hidden confounding factors which we did not take into consideration in the analysis. Potential confounders for which we were unable to adjust in the analysis, due to a lack of data, include distance of the patients' homes from the clinic, psychosocial characteristics and health prognosticators such as CD4. There may thus be some residual confounding in the estimated risk of attrition associated with CAG status and the other factors (age, sex, facility type) that we considered in the analysis. 
Table 3 RIC from the time of eligibility to join a CAG, and factors associated with retention in care, among 2406 patients on ART, between 2008 and 2012, in Tete, Mozambique

\begin{tabular}{|c|c|c|c|c|}
\hline Characteristic & $\begin{array}{l}\text { 12-month RIC } \\
\%(95 \% \mathrm{Cl})\end{array}$ & $\begin{array}{l}\text { 24-month RIC } \\
\%(95 \% \mathrm{Cl})\end{array}$ & $\begin{array}{l}\text { HR } \\
(95 \% \text { Cl) }\end{array}$ & $\begin{array}{l}\text { aHR } \\
(95 \% \mathrm{Cl})\end{array}$ \\
\hline All $(n=2406)$ & 90.8 (89.5 to 92.0$)$ & 86.0 (84.2 to 87.6$)$ & - & - \\
\hline \multicolumn{5}{|l|}{ CAG status* } \\
\hline Not in a CAG $(n=2406)$ & 89.5 (87.9 to 90.8$)$ & 82.3 (79.9 to 84.5$)$ & 1.00 (reference) & 1.00 (reference) \\
\hline In a $C A G(n=901)$ & 99.1 (97.3 to 99.7$)$ & 97.5 (95.4 to 98.6$)$ & 0.17 (0.10 to 0.28$)$ & $0.18(0.11$ to 0.19$)$ \\
\hline \multicolumn{5}{|l|}{ Age (years) } \\
\hline $15-24(n=371)$ & 87.7 (83.5 to 90.9$)$ & 81.4 (75.9 to 85.8$)$ & 1.52 (1.09 to 2.11$)$ & 1.65 (1.17 to 2.32$)$ \\
\hline $25-29(n=515)$ & 92.7 (89.9 to 94.7$)$ & 87.1 (83.0 to 90.2$)$ & 0.98 (0.71 to 1.36$)$ & $1.04(0.75$ to 1.45$)$ \\
\hline $30-39(n=945)$ & 90.8 (88.6 to 92.6$)$ & 87.3 (84.6 to 89.6$)$ & 1.00 (reference) & 1.00 (reference) \\
\hline $40-59(n=575)$ & 91.2 (88.3 to 93.4$)$ & 85.8 (82.0 to 88.8$)$ & 1.09 (0.80 to 1.49$)$ & $0.98(0.72$ to 1.34$)$ \\
\hline \multicolumn{5}{|l|}{ Sex } \\
\hline Female $(n=1514)$ & 92.4 (90.8 to 93.7$)$ & 88.9 (86.9 to 90.7$)$ & 1.00 (reference) & 1.00 (reference) \\
\hline Male $(n=854)$ & 88.2 (85.6 to 90.3$)$ & 80.8 (77.4 to 83.8$)$ & 1.78 (1.41 to 2.26$)$ & $1.82(1.42$ to 2.33$)$ \\
\hline \multicolumn{5}{|l|}{ Facility type } \\
\hline Periurban $(\mathrm{n}=960)$ & 90.9 (89.2 to 92.3$)$ & $85.6(83.3$ to 87.7$)$ & 1.00 (reference) & 1.00 (reference) \\
\hline Rural $(n=1446)$ & 90.8 (88.5 to 92.6$)$ & 86.6 (83.7 to 89.0$)$ & 0.97 (0.76 to 1.25$)$ & 0.90 (0.70 to 1.16$)$ \\
\hline \multicolumn{5}{|l|}{ Cohort $\dagger$} \\
\hline $2008(n=148)$ & 93.1 (87.5 to 96.2$)$ & 88.0 (81.4 to 92.4$)$ & 1.00 (reference) & - \\
\hline January-June 2009 (n=229) & 92.5 (88.1 to 95.2$)$ & 85.1 (79.7 to 89.2) & 1.27 (0.77 to 2.10$)$ & - \\
\hline July-December 2009 ( $n=389)$ & 93.7 (90.8 to 95.7 ) & 88.8 (85.1 to 91.6$)$ & 0.94 (0.57 to 1.56$)$ & - \\
\hline January-June 2010 (n=352) & 92.4 (89.0 to 94.8$)$ & 87.4 (83.3 to 90.5$)$ & 1.06 (0.63 to 1.78$)$ & - \\
\hline July-December 2010 ( $n=382)$ & 92.2 (88.9 to 94.5$)$ & 84.4 (80.2 to 87.8$)$ & 0.95 (0.55 to 1.64$)$ & - \\
\hline
\end{tabular}

${ }^{*} \mathrm{CAG}$ status was a time-dependent variable. Patients were in the 'not in CAG' group until they joined a CAG.

†Cohorts were defined as the semesters of each year within the study period (restricted to 2010 to allow for at least 12 months follow-up), and patients were categorised into each cohort by date at which they became eligible for the study (ie, date at which they reached 6 months on ART). The multivariable Cox regression was stratified by cohort, so aHR's were not determined.

HRs were adjusted for the other variables shown, and stratified by calendar cohort in 6 month categories.

aHR, adjusted HR; ART, antiretroviral therapy; CAG, Community ART Group; RIC, retention-in-care.

Moreover, due to the very nature of the CAG model as described in table 1 , ascertainment of being LTFU was likely more accurate among those in CAG compared with those who remained in standard, individual care, which may have resulted in measurement bias. Finally, we were unable to use viral suppression as an outcome because routine viral load monitoring was not available during the study period. Although we found high RIC among patients in CAGs, we were unable to assess adherence to treatment. Further research is needed to compare viral load outcomes of patients in CAGs and patients in individual care.

The finding that attrition may be reduced by a patient-driven ART distribution model has important implications, especially in rural contexts. The high RIC among patients who joined CAGs can be attributed to a combination of factors including: a reduced time spent travelling to and from the facility and queuing at the facility; reduced health care-related transport costs and enhanced information sharing within the community and between the community and healthcare workers. ${ }^{15}$ Peer support and higher levels of self-efficacy have been identified as important enablers of successful lifelong HIV care. ${ }^{27}$ Peer support enhances use of healthcare services and has a positive effect on quality of life. ${ }^{28}$ Rasschaert $e t$ al found that relationships between patients and healthcare providers changed profoundly after the CAG model was implemented. CAG members were perceived by clinic and community staff as coproviders because they took responsibility for medical tasks, served as a channel of communication between community members and healthcare providers and reduced the workload of healthcare workers, especially in rural health facilities. ${ }^{29}$

In 2008, when the CAG model was introduced, clinicians and healthcare workers were concerned about whether medical tasks such as ART distribution could be delegated to patients. The results of this study confirm that ART distribution can be delegated to patients and demonstrates that patients can take responsibility for their lifelong HIV care, especially when supported by their peers. Earlier studies have shown the benefit of involving patients in peer-to-peer activities without remuneration, 
including counselling, tracing of patients LTFU, administrative tasks in health facilities and income-generation projects. ${ }^{30} 31$ But none of these community-based ART delivery models was driven by the voluntary engagement of PLHIV, motivated by their own health needs. Other community-based ART delivery models in Uganda and Kenya have introduced ART delivery to patients' homes by paid lay healthcare workers, who are recognised and accountable as formal healthcare workers and equipped with motorbikes and cell phones. ${ }^{32-34}$

To achieve and sustain high ART coverage, health programme need to differentiate and adapt to the specific needs of different subgroups, including virologically suppressed patients on ART, clinically unstable patients, $\mathrm{HIV} /$ tuberculosis coinfected patients and adolescents. ${ }^{3536}$ For those stable on ART, less frequent clinic visits and out-of-clinic ART refill are recommended to reduce maximally the burdens on patients and rationalise the use of the scarce health workforce. ${ }^{37}$

Currently, CAG are rolled out nationally in Mozambique and in neighbouring countries such as Lesotho, Zimbabwe and Malawi. ${ }^{24}{ }^{25}$ In Tete Province, the daily management of CAG strongly depended of facility-based lay counsellors. ${ }^{15} 29$ Adaptation of this patient-driven delivery model, which was rooted in the rural community of central Mozambique, will be needed to be adapted to local contexts, needs of specific patient groups, available resources and national policies.

\section{CONCLUSION}

RIC was substantially higher among patients on ART in CAG than among those in individual care. Exclusion of the first 6 months on ART from the follow-up period and the exclusion of patients who had been on ART for more than 6 months at the time that CAGs became available at the facility that they were attending reduced the potential for survival bias but, as the study was observational in design, residual or unmeasured confounders may have contributed to the differences observed. Nevertheless, this study confirms that patient-driven ART distribution through CAGs results in high RIC and supports the Mozambique Ministry of Health in rolling out CAG nationally.

Contributors $\mathrm{BT}, \mathrm{TE}, \mathrm{CM}, \mathrm{CDD}$ and TD conceived the study and designed the study protocol. NDS, SD, MJ collected the data. Data analysis was conducted by CM, RW and TD. TD wrote the first draft of the paper. All coauthors contributed to the subsequent draft and approved the final version.

\section{Competing interests None declared.}

Ethics approval This study was approved by the Ethics Review Board of Médecins Sans Frontières (Geneva, Switzerland) and the Mozambican National Bioethics Committee (Comite Nacional De Bioetica para a Saúde).

Provenance and peer review Not commissioned; externally peer reviewed.

Data sharing statement All the available collected data were included in the study. The dataset can be made available on demand.

Open Access This is an Open Access article distributed in accordance with the Creative Commons Attribution Non Commercial (CC BY-NC 4.0) license, which permits others to distribute, remix, adapt, build upon this work non-commercially, and license their derivative works on different terms, provided the original work is properly cited and the use is non-commercial. See: http://creativecommons.org/ licenses/by-nc/4.0/

(c) Article author(s) (or their employer(s) unless otherwise stated in the text of the article) 2017. All rights reserved. No commercial use is permitted unless otherwise expressly granted.

\section{REFERENCES}

1. UNAIDS. Fact sheet 2016. 2016: UNAIDS. http://www.unaids.org/en/ resources/fact-sheet.

2. UNAIDS. 90-90-90: an ambitious treatment target to help end the AIDS epidemic. Switzerland, Geneva: UNAIDS, 2014. http://www. unaids.org/sites/default/files/media_asset/90-90-90_en_0.pdf.

3. World Health Organization. Supplement to the 2013 consolidated guidelines on the use of antiretroviral drugs for treating and preventing HIV infection. recommendations for a public health approach: World Health Organization, 2014. http://www.who.int/hiv/ pub/guidelines/arv2013/arvs2013upplement_march2014/en/.

4. Fox MP, Rosen S. Retention of adult patients on antiretroviral therapy in low- and Middle-Income countries: systematic review and meta-analysis 2008-2013. J Acquir Immune Defic Syndr 2015;69:98-108.

5. Govindasamy D, Ford N, Kranzer K. Risk factors, barriers and facilitators for linkage to antiretroviral therapy care: a systematic review. AIDS 2012;26:2059-67.

6. Kredo T, Ford N, Adeniyi FB, et al. Decentralising HIV treatment in lower- and middle-income countries. Cochrane Database Syst Rev 2013;6:CD009987.

7. Callaghan M, Ford N, Schneider H. A systematic review of taskshifting for HIV treatment and care in Africa. Hum Resour Health 2010;8:8:8

8. Duncombe C, Rosenblum S, Hellmann N, et al. Reframing HIV care: putting people at the centre of antiretroviral delivery. Trop Med Int Health 2015;20:430-47.

9. Lambdin BH, Micek MA, Sherr K, et al. Integration of HIV care and treatment in primary health care centers and patient retention in central Mozambique: a retrospective cohort study. J Acquir Immune Defic Syndr 2013;62:e146-e152.

10. Wandeler G, Keiser O, Pfeiffer K, et al. Outcomes of antiretroviral treatment programs in rural Southern Africa. J Acquir Immune Defic Syndr 2012;59:e9-e16.

11. Caluwaerts $\mathrm{C}$, Maendaenda R, Maldonado F, et al. Risk factors and true outcomes for lost to follow-up individuals in an antiretroviral treatment programme in Tete, Mozambique. Int Health 2009;1:97-101.

12. Posse $M$, Baltussen R. Barriers to access to antiretroviral treatment in Mozambique, as perceived by patients and health workers in urban and rural settings. AIDS Patient Care STDS 2009;23:867-75.

13. Kober K, Van Damme W. Expert patients and AIDS care. A literature review on expert patient programmes in high-income countries, and an exploration of their relevance for HIV/AIDS care in low-income countries with severe human resource shortages. $2008 \mathrm{http} / / / \mathrm{www}$. eldis.org/fulltext/kober-vandamme.pdf.

14. Bemelmans M, Baert S, Goemaere E, et al. Community-supported models of care for people on HIV treatment in sub-Saharan Africa. Trop Med Int Health 2014;19:968-77.

15. Rasschaert $F$, Telfer $B$, Lessitala $F$, et al. A qualitative assessment of a community antiretroviral therapy group model in Tete, Mozambique. PLoS One 2014;9:e91544.

16. Decroo T, Telfer B, Biot M, et al. Distribution of antiretroviral treatment through self-forming groups of patients in Tete Province, Mozambique. J Acquir Immune Defic Syndr 2011;56:e39-e44.

17. Decroo T, Koole O, Remartinez D, et al. Four-year retention and risk factors for attrition among members of community ART groups in Tete, Mozambique. Trop Med Int Health 2014;19:514-21.

18. World Health Organization. WHO Country Cooperation Strategy 2009-2013. Mozambique. World Health Organization 2009 http:// www.who.int/countryfocus/cooperation_strategy/ccs_moz_en.pdf.

19. UNAIDS. AIDSinfo: UNAIDS, 2016. Available at. http://aidsinfo. unaids.org/.

20. Ministry of Health Mozambique. Global AIDS Response Progress Report for the Period 2010 - 2011, Mozambique: Ministry of Health Mozambique, 2012. http://www.unaids.org/sites/default/files/ country/documents//file,94670,es.pdf.

21. Decroo T, Panunzi I, das Dores $C$, et al. Lessons learned during down referral of antiretroviral treatment in Tete, Mozambique. J Int AIDS Soc 2009;12:6. 
22. Lawn SD, Harries AD, Anglaret $X$, et al. Early mortality among adults accessing antiretroviral treatment programmes in sub-Saharan Africa. AIDS 2008;22:1897-908.

23. Gupta A, Nadkarni G, Yang WT, et al. Early mortality in adults initiating antiretroviral therapy (ART) in low- and middle-income countries (LMIC): a systematic review and meta-analysis. PLOS One 2011;6:e28691.

24. Jobarteh K, Shiraishi RW, Malimane I, et al. Community ART support groups in Mozambique: the potential of patients as partners in Care. PLoS One 2016;11:e0166444.

25. Vandendyck M, Motsamai M, Mubanga M, et al. Community-based ART resulted in excellent retention and can leverage community empowerment in rural Lesotho: a mixed method study. HIVIAIDS Research and Treatment - Open Journal 2015;2:44-50.

26. Wandeler G, Keiser O, Pfeiffer K, et al. Outcomes of antiretroviral treatment programs in rural Southern Africa. J Acquir Immune Defic Syndr 2012;59:e9-e16.

27. Langebeek N, Gisolf EH, Reiss P, et al. Predictors and correlates of adherence to combination antiretroviral therapy (ART) for chronic HIV infection: a meta-analysis. BMC Med 2014;12:142.

28. Bateganya $\mathrm{MH}$, Amanyeiwe $\mathrm{U}$, Roxo $\mathrm{U}$, et al. Impact of support groups for people living with HIV on clinical outcomes: a systematic review of the literature. J Acquir Immune Defic Syndr 2015;68(S3) :S36874.

29. Rasschaert F, Decroo T, Remartinez D, et al. Sustainability of a community-based anti-retroviral care delivery model-a qualitative research study in Tete, Mozambique. J Int AIDS Soc 2014;17:18910.
30. Wouters E, Van Damme W, van Rensburg D, et al. Impact of baseline health and community support on antiretroviral treatment outcomes in HIV patients in South Africa. AIDS 2008;22:2545-8.

31. Zachariah R, Teck R, Buhendwa L, et al. How can the community contribute in the fight against HIV/AIDS and tuberculosis? An example from a rural district in Malawi. Trans $R$ Soc Trop Med Hyg 2006;100:167-75

32. Weidle PJ, Wamai N, Solberg P, et al. Adherence to antiretroviral therapy in a home-based AIDS care programme in rural Uganda. Lancet 2006;368:1587-94.

33. Jaffar S, Amuron B, Foster S, et al. Rates of virological failure in patients treated in a home-based versus a facility-based HIV-care model in Jinja, southeast Uganda: a cluster-randomised equivalence trial. Lancet 2009;374:2080-9.

34. Wools-Kaloustian KK, Sidle JE, Selke HM, et al. A model for extending antiretroviral care beyond the rural health centre. $J$ Int AIDS Soc 2009;12:22.

35. Grimsrud A, Bygrave H, Doherty M, et al. Reimagining HIV service delivery: the role of differentiated care from prevention to suppression. J Int AIDS Soc 2016;19:21484.

36. Phillips A, Shroufi A, Vojnov L, et al. Sustainable HIV treatment in Africa through viral-load-informed differentiated care. Nature 2015;528:S68-S76.

37. World Health Organization. Factsheet. HIV treatment and care.What's new in service delivery? World Health Organization, 2015. http:// apps.who.int/iris/bitstream/10665/204461/1/WHO_HIV_2015.46 eng.pdf?ua $=1$. 